Schweiz. Z. Path. Bakt. 1958;21:124-131

\title{
Allocution
}

\section{J. Delarue}

President de la Société Internationale de Pathologie Géographique.

Monsieur le Représentant du Secretaire d'Etat à la Santé Publique et à la Population,

Mes collègues me pardonneront de m'adresser à vous avant de leur souhaiter une cordiale

bienvenue. Votre presence ici, comme représentant de Monsieur le Directeur General de la Santé Publique, empêché par des motifs bien légitimes que nous déplorons profondément, signifie que, en France comme aílleurs, le Gou-vernement s'intéresse à notre activité. Soyez-en vivement remercié.

Monsieur le Doyen de la Faculté de Médecine de Paris,

Vous voulez bien nous accueillir ici chez vous, dans ces vieux murs deux fois séculaires. C'était

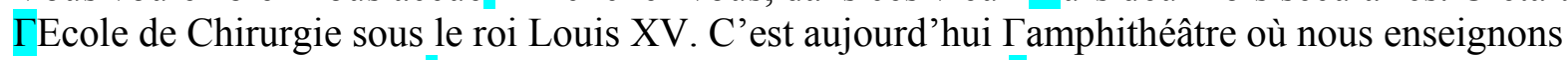
et c'est aussi le lieu de colloques internationaux. Vous êtes present ce matin parce que rien d'utile ne peut se dérouler dans cette Faculté sans que vous ayez à cæur de le favoriser et d'y présider, et aussi parce que vous attachez beaucoup de prix à la venue parmi nous de savants de pays amis qui sont vos hôtes. En leur nom et en mon nom personnel, je vous exprime une vive gratitude.

Mesdames,

Mes chers collègues,

En vous voyant si nombreux ce matin, je pense que la Sixième Conference de la Société Internationale de Pathologie Géographique s'ouvre sous d'heureux auspices et que, comme les précé-dentes, elle sera Toccasion de confrontations utiles et d'un travail fructueux. Je vous souhaite la bienvenue dans cette cite, que vous nous avez fait Thonneur de choisir pour y tenir vos assises.

C'est le lieu de naissance de notre Société, ce que beaucoup d'entre vous ignorent peut-être. En 1929, une reunion plénière de la Société Anatomique de Paris, organisée par Gustave Roussy, avait entendu $\Gamma$ expose de deux rapports sur les cirrhoses du foie : $\Gamma$ un de Robert Ræssle, et $\Gamma$ autre de Noel Fíessínger. Les points de vue et les conclusions relatifs à $\Gamma$ étiologie et aux caractères ana-

D e 1 a $\gamma$ u e , Allocution

125

tomo-pathologiques des cirrhoses se sont révélés très dissemblables au cours de la discussion de ces rapports. On s'est alors aperçu que les maladies pouvaient être différentes selon les pays. L'idée d'une pathologie géographique est ainsi née dans Гesprit d'Aska-nazy, de L. Aschoff, de Josselín de Jong, de Gustave Roussy. Notre groupement a été fondé sur la base Internationale d'abord réduite, puis élargie, qui est sa raison d'etre. On s'est réuni à Geneve, puis à Utrecht, puis à Stockholm, une quatrième fois dans la «bonne ville » de Liège avec Taimable hospitalité du Professeur Jean Firket, et, il y a trois ans, dans la belle cite de Washington sous la présidence du Professeur Howard T. Karsner et la direction pleine de bonne grace du Professeur Robert A. Moore. Quinze années de silence ont séparé la Conference de Stockholm de celle de Liège. La 
guerre était venue, pendant laquelle on s'intéressait sans doute à la géographie, mais moins à la pathologie spontanée de tous les pays dans le dessein d'y porter remède. Avec tant d'autres choses, notre jeune Société faillit sombrer dans cette tourmente. Lorsque $\Gamma$ on put s'aviser que les nations étaient à même d'échanger, non plus des bombes mais des documents scientifiques sur les divers aspects des maux dont souffre Thumanité, il a fallu la généreuse initiative et Гopiniâtreté de trois hommes pour ranimer ou allumer dans le monde la flamme de la Pathologie Géographique. Ce furent le Professeur Loitís Míchaud, de Lausanne, le Professeur Folke Henschen, de Stockholm, et le Professeur Frederic Roulet, de Bale.

Louis Míchaud n'est plus. Notre Société, qui sait ce qu'elle doit à son action, en avait fait son President d'Honneur en 1952 ; elle porte aujourd'hui son deuil. Louis Míchaud est né à Berne en 1880 , d'un père bourguignon et d'une mere polonaise. De $\Gamma$ un il avait hérité $\Gamma$ équilibre, la perseverance dans $\Gamma$ effort et Tironie souriante que $\Gamma$ on trouve chez les terriens de Vézelay ou de Cha-gny ; de Гautre il tenait Гaffectivité d'un Stanislas Leszczyýskí et la sensibilité d'un Chopin, et un gout très sûr des arts plastiques. III y a aussi une génétique géographique. Cette conjonction de races avait créé un homme exceptionnellement doué, aux idées fécondes, à Tesprit universel. Après des etudes médicales à Berne, où il fut $\Gamma$ assistant de Sahli, puis de Langhans, Míchaud fut attire par les grands cliniciens allemands de Гépoque. II resta de longues années auprès de Krehl, à Heidelberg, puis de Luthje à Francfort. En 1913, à Гâge de 33 ans, il fut nommé Professeur de clinique médi-cale à Lausanne en remplacement de Bourget. II devait occuper

126

D e 1 a $\gamma$ u e , Allocution

cette chaire pendant 37 années, travaillant sans relâche, créant de nouvelles méthodes d'exploration médicale, formant à son exemple de nombreux disciples. Louis Michaud était un médecin interniste. Mais il était aussi un biologiste, et aussi un pathologiste très averti, qui savait donner la réplique à son ami Askanazy. Ses travaux scientifiques, qui sont nombreux, et intéressent notamment $\Gamma$ histo-logie du goitre, le métabolisme des protéines, $\Gamma$ hyperinsulinisme, Гulcère gastrique, $\Gamma$ hyperchlorémie, precedent de cette triple tendance. Louis Michaud voyageait beaucoup. II avait une large audience dans beaucoup de pays. G'est sans doute Tétendue de son information et sa largeur de vues qui lui avaient fait comprendre Tintérêt des questions qui nous occupent. II a été $\Gamma$ un des meilleurs animateurs de notre Société. Nous garderons pieusement le souvenir de notre President d'Honneur.

Un autre grand promoteur de la Pathologie Géographique a disparu depuis notre dernière reunion. Robert Ræssle est mort il y a quelques mois, à $\Gamma$ âge de 80 ans. Qu'il me soit permis d'évo-quer ici la mémoire de ce grand pathologiste. Né à Augsbourg, étudiant en médecine à Munich, Ræssle a été dans cette ville Гas-sistant de Heller, puis de Hertwig. Bientôt il devient le principal collaborateur de Bollínger, auprès de Max Borst. En 1911, il est nommé Professeu ordinaire dans le nouvel institut de Гuniversité d'léna. L'année 1921 le conduit à Bale, où il demeure huit années, jusqu'à son accession à la premiere place de la pathologie alle-mande, la chaire de Rudolf Virchow à Berlin. Au cours de sa longue vie, tout entière consacrée à la recherche et à $\Gamma$ enseigne-ment, Ræssle a fondé une prestigieuse et grande école, dont se réclament aujourd'hui beaucoup de nos collègues. La tradition de Zenker qu'il tenait de Heller, et celle de Virchow qu'il devait à Bollinger avait abouti chez lui à Theureuse synthèse intellectuelle dont procède son ceuvre scientifique. Celle-ci est très importante, et $\Gamma$ on peut être certain qu'elle est durable. Dois-je rappeler ici ses beaux travaux sur Гinflammation, sur Гexpression morphologique de Гallergie, sur les granulomes spécifiques ? Ai-je besoin de citer 
ses etudes sur ce qu'il a appelé la « pathologie de constitution », veritable Anatomie pathologique de la Famille, et sur la pathologie du vieillissement? En pathologie spéciale, on sait Timportante contribution qu'il a fournie à la connaissance des maladies du foie. Conduisant ses recherches, sa science, sa technique, avec une precision rigoureuse et réellement mathematique, Ræssle se laissait

Delarue, Allocution

127

aller aussi à philosopher lorsqu'il commentait les faits observes. Comme $\Gamma$ a écrit $\Gamma$ un de ses

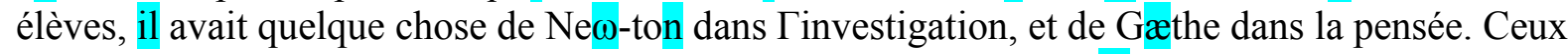
qui Гont approché et entendu n'ont pas oublié la distinction de son main-tien, la clarté de ses exposes et $\Gamma$ élégance de son discours. Ils savent aussi quel courage il a montré dans $\Gamma a d v e r s i t e ́$, au cours d'une grave maladie et devant la ruine de son cher et grand insti-tut. Robert Ræssle a été Tun des plus éminents pathologistes de ce siècle. II tient une grande place dans le cæur de ses disciples. II occupera longtemps $\Gamma$ esprit de tous les pathologistes du monde.

On constate avec mélancolie que, revenant à Paris vingt-cinq ans après y avoir été enfantée, notre Société se trouve aujourd'hui privée de ceux qui lui ont donné le jour. Qu'il me soit permis en cet instant d'avoir une pensée particulière, une pensée filiale pour la grande mémoire de mon maître Gustave Roussy, qui aurait été si heureux de vous accueillir dans cette Université de Paris, qu'il dirigeait. Mais notre reunion démontre que $\Gamma æ u v r e$ survit à ses auteurs et que la pérennité en est assurée. La Société Internationale de Pathologie Géographique est bien vivante, avec une âme bien trempée. Nous avons le bonheur d'avoir pour guides nos deux doyens, nos deux « Sages », le Professeur Howard T. Karsner et le Professeur Folke Henschen, Гun et Гautre pionniers de notre science. Qu'ils me permettent de leur exprimer publiquement notre affection respectueuse. Nous avons aussi la chance d'avoir un gouvernement stable, ce qui passe pour être un bienfait. D'une main ferme, le Professeur F. C. Roulet exerce à lui seul le pouvoir exécutif. II est permanent, aux côtés de presidents éphémères, obligatoirement déchus dès quills ont une fois rempli leurs functions. II est le pivot de notre organisation. Pour accomplir si bien sa lourde tâche, il ne menage ni son temps, ni sa peine. Je suis sûr d'etre votre interprète en lui disant notre reconnaissance.

Mais le système comporte quelques imperfections que ma situation présente me donne le pouvoir de signaler sans risquer d'etre immédiatement contredit. Le Secretaire general, et le Co-mité directeur sûr lequel il s'appuie, représentent en réalité un gouvernement et un parlement « fantoches ", car ils n'ont personne à gouverner. Ils sont élus, mais leurs électeurs s'évanouissent dès qu'ils ont vote. Nous sommes une société sans sociétaires, une tête sans corps et un corps sans membres. Notre groupement ne se manifeste que par des paroxysmes transitoires tous les trois ans,

128

D e 1 a ru e, Allocution

sous la forme de conferences dont les participants, recrutés selon les sujets traités, ont $\Gamma$ insigne honneur d'etre membres de la So-ciété de Pathologie Géographique, mais pour trois jours seulement... Nous aurons à vous proposer, demain, à vous assemblée constituante, d'adopter de nouveaux statuts. Ils veulent nous per-mettre des etudes plus longues, plus completes, dont un effort conti-nu, réparti entre beaucoup d'enquêteurs, est la condition essentielle.

Je reprendrai d'autre part à mon compte les pertinentes critiques que nous a adressées à Liège, en 1952, le Docteur Jacques M. May. Du fait des origines et des tendances de la plupart d'entre nous, nous nous sommes beaucoup intéressés à la Pathologie, et moins à la Géographie. A 
propos de quelques maladies déjà, nos conferences ont été $\Gamma$ occasion d'etudes de grande qualité et vrai-ment exhaustives. Les documents sur lesquels elles ont été basées, venus de plusieurs continents, leur ont conféré sans doute un caractère international. Mais la repartition de ces diverses maladies dans le monde, c'est-à-dire leur distribution géographique, n'a pas été mise nettement en evidence, ceci faute de coordinations adéquates et de méthodes de travail efficaces. A cet état de choses, on peut remédier. Le Docteur May a formulé depuis quelques années d'utiles propositions. Hier, au cours d'une reunion organisée ici-même par le Conseil des Organisations Internationales des Sciences Médicales en prelude à cette Conference, on a recherche les moyens propres à nous fournir les données dont nous avons besoin pour remplir notre mission. Je suis sûr que ces efforts, conjugués avec les nôtres, pourront aboutir, et je le souhaite vivement.

Nos buts sont en effet clairs et bien définis. II importe de les rappeler ici pour ceux qui n'en sont pas exactement informés. Nous nous proposons de savoir si les lesions qui caractérisent les maladies sont les mêmes dans tous les pays où celles-ci sont ob-servées. Nous sommes des pathologistes, en effet, et nous pensons que cet aspect lésionnel de $\Gamma$ état morbide en est encore la plus exacte et la plus constante traduction. Mais la pathologie a pour nous un large sens. Elle n'est plus depuis longtemps la morpho-logie pure qui sufñsait à nos lointains devanciers. Elle suppose la connaissance des manifestations cliniques qui traduisent les lesions; elle conduit à Гidentification des perturbations biologi-ques qui engendrent celles-ci et les suivent. Surtout, elle suggère la recherche des causes.

D e 1 a $\gamma$ u e , Allocution

129

Constater des differences entre les cirrhoses hépatiques de ГEurope et celles de la Malaisie ou de ГAfrique Noire conduit immédiatement à chercher les raisons de ces dissemblances. C'est par là que notre Société de Pathologie est géographique. Pour com-prendre le role des facteurs étiologiques d'une maladie, il faut savoir sous quels aspects cliniques et anatomiques elle se présente dans toutes les contrées de notre planète et quelle en est la relative frequence dans chacune d'elles par rapport au chiffre de la population. Ceci conduit à rechercher le role possible de facteurs raciaux et héréditaires innés, du climat, de $\Gamma$ habitat, de $\Gamma$ alimen-tation, d'éventuelles infections ou intoxications exogènes associées, de Гactivité physique, des preoccupations et des servitudes même des populations, et de ces us et coutumes dont Montaigne se plai-sait à souligner la diversité parmi les hommes.

III n’y a pas une affection dont Гétude ne puisse bénéficier de telles enquêtes. Les maladies infectieuses même, dont $\Gamma$ agent pa-thogène est parfaitement identifié, sont conditionnées par des facteurs « environnants » ou intrinsèques trop souvent méconnus. Mais nous abordons dans cette Société les plus difficiles problè-mes. Nous nous attachons surtout à $\Gamma$ examen de maladies répan-dues sur tout le globe et qualifiées de « constitutionnelles » par ceux qui renoncent à en préciser les causes complexes. Nous les analysons avec $\Gamma$ espoir de lever tin peu le voile de mystère qui enveloppe leur origine. Là où les raisonnements les plus subtils, où les recherches expérimentales les plus precises ont échoué, la Pathologie géographique pourra peut-être réussir. L'intérêt de telles prospections ne peut échapper à personne. Elles tendent tout d'abord à satisfaire ce demon de curiosite que tout homme de science porte en soi, et peuvent accroître nos con-naissances sur la pathogénie des états morbides. Mais il s'en faut qu'elles soient conduites dans un but purement spéculatif. Elles n'ont pas seulement pour effet d'enrichir notre patrimoine scien-tifique commun. A propos de certains états pathologiques, nos recherches bien conduites nous permettront de sortir du domaine de Thypothèse pour obtenir une certitude. Nous saurons 
que telle affection est certainement favorisée par de mauvaises conditions d'habitat, que telle autre résulte d'une alimentation excédentaire, parce qu'elle se voit dans les pays « où coule le beurre et le miel » que détestait Zarathoustra, qu'une troisième est engendrée par le douloureux, mais irresistible développement du machinisme. Sûrs

130

Delarue, Allocution

de nos conclusions éminemment valables de pathologistes, nous en ferons part aux hygiénistes, aux économistes et aux pouvoirs publics. « La cirrhose du foie s'observe chez les sujets sous-alimentés. Donnez à manger à ceux qui ont faim. »- « Mais elle se voit aussi chez ceux qui ont trop soif. De grace, débarrassez-nous de Гalcoolisme. »

Notre Société peut à bon droit revendiquer une place parmi les sources d'information et les instruments de ГOrganisation Mondiale de la Santé. Je pense qu'elle peut efficacement participer au combat pour le bien-être et la santé des hommes. C'est sans doute pour cette raison que nous pouvons saluer ici la presence du Docteur Yves Bíraud, Directeur des Services d'Epidémiologie et des Statistiques Sanitaires à ГO.M.S.

Je suis sûr, en tout cas, que la Pathologie géographique est un grand bienfait intellectuel pour ceux qui la pratiquent, et ce n'est pas là la moindre de ses vertus. La Fontaine disait plaisamment qu'un mollusque entr'ouvrant sa coquille sait se donner un horizon plus large que les hommes replies sur eux-mêmes, pré-occupés seulement de leurs petits intérêts personnels et de leur vie quotidienne. Avec la Pathologie géographique, nous ouvrons notre coquille, nous voyons la pathologie non plus seulement dans nos instituts, dans nos villes, dans nos pays respectifs. Nous la sentons et la pensons à $\Gamma$ échelle mondiale. Cette mole hydati-forme, peu fréquente ici, se peut-il qu'elle soit si souvent obser-vée en Extreme-Orient, et pourquoi ? Ce cancer primaire du foie, encore assez rare en Europe, comment est-il si répandu ailleurs, par exemple en Afrique Noire? Nous sentons bien que c'est un esprit nouveau qui nous anime ainsi. On se prend à se sentir en quelque sorte solidaire de tous les hommes de cette planète, et $\Gamma$ on ne s'étonne plus, comme le héros de Montesquieu, que Ton puisse être Persan. A la dernière Conference Internationale de la Tuberculose, à la Nouvelle-Delhi, le Pandit Nehru, premier minis-tre de Tlnde, a vanté et prôné, en de nobles paroles, $\Gamma$ esprit uni-versel. II $\Gamma$ a reconnu chez beaucoup de médecins et d'hommes de science, et formulé le væu qu'il s'impose par contagion à ceux qu'il a appelés les gens de son metier, c'est-à-dire aux politiciens. Nous revendiquons pour nous cet esprit universal, car il est notre raison d'etre. Je souhaite, avec le Pandit Nehru, qu'il devienne une mode irresistible pour abaisser les barrières, et lever les ri-deaux.

De 1 a $\gamma$ u e, Allocution

131

Par votre presence ici, vous démontrez, mes chers Collègues, que vous en êtes animés. Beaucoup d'entre vous viennent de loin-tains pays pour en témoigner. Diderot a mis cinquante-trois jours pour se rendre de Paris à St-Pétersbourg aupГès de la Grande Catherine. En quelques heures, on survole aujourd'hui d'immen-ses continents et de larges oceans. Mais le mérite des grands voyageurs ne s'est pas réduit pour autant. Pour avoir le droit de don-ne $\Gamma$ ce rapide coup d'aile, il faut parfois plusieurs mois d'allées et venues préparatoires de bureau en bureau dans une même petite cite. Je sais que quelques-uns de nos collègues, désireux depuis longtemps de venir ici, sont encore à cette heure perdus dans $\Gamma$ im-mense labyrinthe des offices de police et de finances. Ceci nous prive de leur presence, et nous le regrettons vivement.

Vous êtes venus pour apporter ou synthétiser des faits d'obser-vation mondiale sur $\Gamma$ une de ces maladies dont $\Gamma$ étude répond exactement aux desseins de notre Société. Nous avons hate d'en 
profiter, et il est temps que prenne fin ce discours de bienvenue. II est d'usage, au cours de nos conferences, qu'une discipline stricte soit imposée aux orateurs trop prolixes. A cette servitude, je dois me soumettre le premier et donner Гexemple. Ce n'est pas sans regret, car il me semble que $\mathrm{j}$ 'aurais encore beaucoup de choses à vous dire, beaucoup de compliments à vous faire, beaucoup de professions de foi à articuler, tant est profonde la joie que j'é-prouve de vous voir ici. 11 est aussi habituel que nos reunions se déroulent sous le signe de la simplicité et de la cordialité. Nous respecterons cette tradition, et nous espérons ainsi resserrer les liens qui unissent les hommes et les idées. Enfin, il est constant que les conferences de notre Société laissent un agréable souvenir à leurs participants. Nous voudrions que celle-ci ne soit pas dif-férente de ses devancières. Si cette espérance n'est pas déçue, nous le devrons à ceux qui en ont assure Torganisation, à notre Secretaire general naturellement, et aussi au Docteur Hardouín et au Docteur Paulas. Ce sera aussi grace aux organismes qui ont bien voulu nous aider matériellement, le Ministère des Affaires Etran-gères, le Secretariat d'Etat à la Santé Publique et à la Population, et le C.I.O.M.S.

Mais ce sera surtout grace à votre presence, à votre assiduité, à votre science. Pour vous et surtout par vous, mes chers Collègues, je souhaite le succès de la 6e Conference Internationale de Patho-logie Géographique. 